\title{
A Method for Generating Decision Rules of UAV for Precision Operation
}

\author{
Huifen, Dong ${ }^{1}$, Xiaoli, Wang ${ }^{2}$ and Qingji, Gao ${ }^{1+}$ \\ Institute of Robotics, Civil Aviation University of China, Tianjin, China
}

\begin{abstract}
In order to reduce the complexity of the process decision of UAV operations, a fine and comprehensive operation decision rule is established, and the rough decision rule generation method is studied. Firstly, the design rules of spatial attribute for job requirements are studied, Based on the density distribution of the sample of the precision operating condition attribute, a fine quantization method based on the tangent property is proposed. Then, the attribute reduction based on the rough set attribute set dependency is used to obtain the decision rule after removing the redundant attribute. The rationality and accuracy of the decision rules generation method is verified by the example of "precision agriculture" competition.
\end{abstract}

Keywords: design rules; distribution density; precise quantization; decision making; UAV mission decision

\section{Introduction}

In the decision-making system of UAV operation, the establishment of decision-making method plays an important role in the optimization and realization of UAV operations. Due to the uncertainty of most jobs, the incompleteness and inconsistency of the task information, the difficulty of decision optimization is greatly increased [1]. If the selection of influencing factors and the quantization of the range are not appropriate, the accuracy of the UAV operation will be affected. In the process of generating the decision rules of the job, it is necessary to make a comprehensive evaluation of various attribute factors, select the reasonable attributes, and design a detailed method to quantify the value of attributes, in order to achieve accurate completion of the job.

The general way to solve the decision problem is to establish the algorithm model. As in literature[2], the fuzzy similarity class is introduced, and the fuzzy quantization is carried out. This method takes into account the characteristics of fuzzy attributes, can adapt to the general decision-making situation and problems, but ignores the fine degree of attribute selection, can not guarantee the accuracy of decision-making; As in literature[3], the attribute tree is established, and the decision tree is simplified by the decision-making conference, this method takes into account the problem of many attributes in the decision process, but it will lead to the weakening of attributes, and the selection of attributes can not cover the whole task change process, and can not achieve the precise conversion of the task process. These optimization methods take into account the uncertainty and fuzziness of attributes, but do not give the choice of attributes in the decision making process, which will increase the complexity of attribute selection.

In literature [4], the continuous attributes are quantified by the method of attribute importance and decision classification. If the condition attribute is the same, and the decision attribute is only, then the attribute values are quantized to a level. The decision table has a high recognition rate after quantization, but the UAV in the precise operation, need to design a fine quantization method in various tasks range, in order to achieve accurate completion of tasks, therefore the value of the property in the range of quantification task becomes a key problem.

Aiming at the above problems, this paper is based on the background of "precision agriculture" of UAV competition. The mission space attribute design rules is studied for the task requirements, and the precise quantification method is designed according to the range of the condition attributes in the precise operation.

\footnotetext{
Corresponding author. Tel.: + 13302003271 .

E-mail address: 1401385025@qq.com.
} 
Attribute reduction algorithm is used to reduce the attributes, to remove redundant attributes, and to give the final reduction results. Then, the rationality and accuracy of the design rules and attribute quantification methods are verified by the example of UAV precision agriculture.

\section{Design of Task Attribute}

The state transition diagram of UAV precision agriculture competition is as shown in Figure 1.

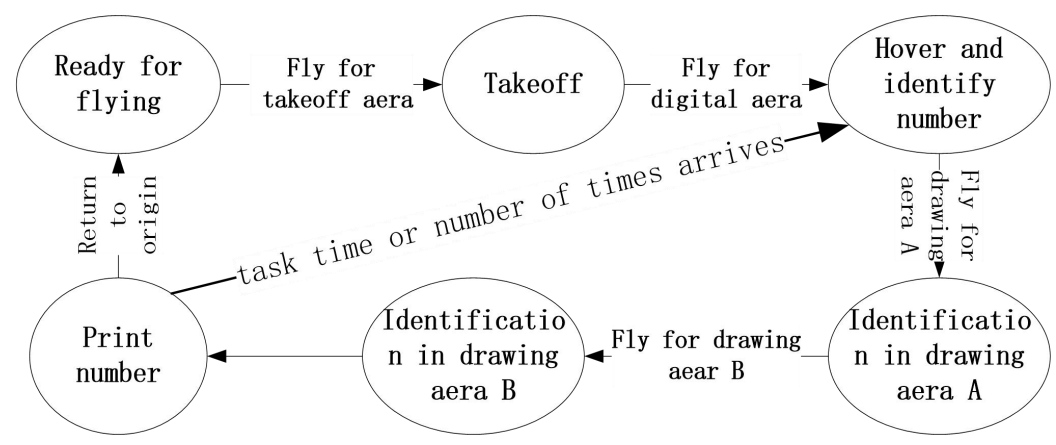

Fig. 1: State transition diagram of precise task.

\subsection{Selection Criteria for Task Condition Attributes}

Based on rough set theory, attribute selection is an essential condition for mission decision making, which is the factor that causes the change of the operation decision after the change of the state or condition. In an operating system, based on the thinking of the control system elements, to select the factors that affect the job decision, according to the formula (1) shown in the decision function to select the condition attribute.

$$
y=f(x, u, T)
$$

In formula(1), y represents the output of decision; $x, u, T$ shows the three aspects the selection of the condition attributes ,among them, $x$ represents state variable of task process; $u$ represents control variable(actuator); $T$ represents state transition variable; $f$ represents the mapping of conditional variables to decision output, i.e., decision rule table

According to the above three aspects, in the UAV operating system, the state variable refers to the UAV position and attitude variables; control variables refer to the UAV to complete actuator operation changes; state transfer refers to the change of state variables.

The pose variables mainly refer to the sensor data, the sensor data includes the original data, the two processing data and the fused data. The sensor in UAV operating system includes camera, GPS and barometer. Image recognition, coordinate position own to raw data of sensor; the credibility of the identification result is the data obtained from the results of multiple identification, belonging to the two processing data; Height belongs to fusion data.

The state transition variable is the change of state. The change of the state refers to the attribute factor of the decision state change, including the time of the execution of the job, which refers to the effective time of the "precise operation"; the number of times of operation execution refers to the "precision operation" The number of spray required; the control variable refers to the change of the operating agency, including whether the spraying operation is completed or not. According to the division of the task state and the conditions of the above influence state transformation, the following 10 conditional variables are selected:

Coordinate position $\left(a_{1}\right)$, height $\left(a_{2}\right)$, time limit condition $\left(a_{3}\right)$, identify digital number or not identify $\left(a_{4}\right)$, identify drawing area number or not identify $\left(a_{5}\right)$, recognition result credibility $\left(a_{6}\right)$, mission completed or not $\left(a_{7}\right)$, spraying time $\left(a_{8}\right)$, mission times $\left(a_{9}\right)$, mission time arrive or not $\left(a_{10}\right)$, history state $\left(a_{11}\right)$.

\subsection{Selection Rules for Job Decision Attributes}


The decision attribute is the control quantity for the UAV to provide the behavior action, which takes the different behavior of the UAV in different areas as the decision attribute. It can guarantee the stability of UAV flight and the accuracy of the operation.

In "precision agriculture" operating system, choosing the behavior of different states in Figure 1 as decision attributes, includes Ready to take off, fly to the point of departure, arrival and take off point area, to digital recognition, digital drawing area to hover $\mathrm{A}$, rendering $\mathrm{A}$ area identification, $\mathrm{B}$, to draw the drawing area of $\mathrm{B}$ recognition, digital printing.

\section{Quantization Design Based on the Property of Tangent Distribution}

Because the task condition variables have continuous attribute values, the quantization of continuous attributes is the first problem to be solved in rough set preprocessing. In the task system, the quantitative design of the attributes is mainly based on the task requirements, task state changes and condition attributes.

Work area consists of two parts: the working section and non working section, Quantitative design is based on the principle that the distribution density of the operating area is large, and the non-operating area is small, a quantization method based on the tangent property is proposed.

The quantization function based on the tangent property:

$$
x^{\prime}=f(x)=k * \arctan (\omega x+\varphi)+b
$$

The quantization function is shown in Figure 2:

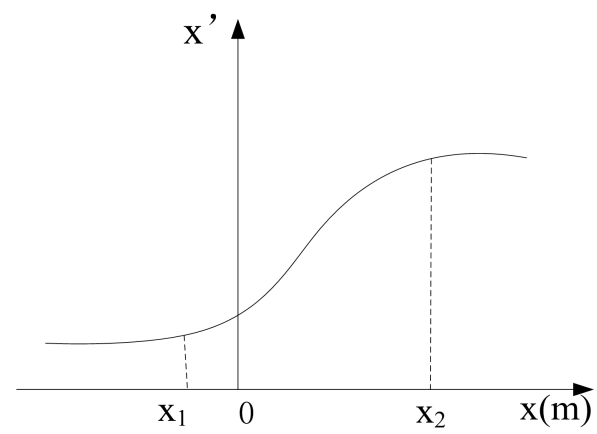

Fig. 2: The curve of quantization function.

In Figure 2, the abscissa is the sample, and the ordinate is the quantized value. The distribution density is defined as the ratio of the number of samples and the range of the sample:

$$
\rho_{d}=d x^{\prime} / d x
$$

The attributes are quantified on the condition of different work area, need for specific operational requirements to adjust four parameters: kappa, Omega, phi and B, which can meet the principle that the distribution density of the operating area is large, and the non operating area is small.

Using the above design method to quantify the design of working area condition attributes .Among them, whether or not to identify the number, whether the completion of the spray, the arrival of the work time is already discrete information, no longer need quantified.

The quantification of recognition confidence is based on the number of the same recognition results, each time to identify the target 10 times, if more than 6 times for the same result, quantified as 1, otherwise quantified as 0 ; Whether or not the arrival time is based on the number of matches in the game, if the time is exceeded, quantified as 1.

Analyze the specific job requirements, according to Figure 2 and the quantification rules to quantify the coordinates of the location.

When the (UAV) is in the working section, the quantitative density of the operating section is large according to the quantization rule, and it is quantized to 2,3,4,5,6,7,8,9. Non-operating sector distribution density is small, coarse quantification, quantification of 0,1 . 
When the height attribute is quantization, according to the demand of the height of the job, the quantization function curve following the quantization is shown in Figure 3.

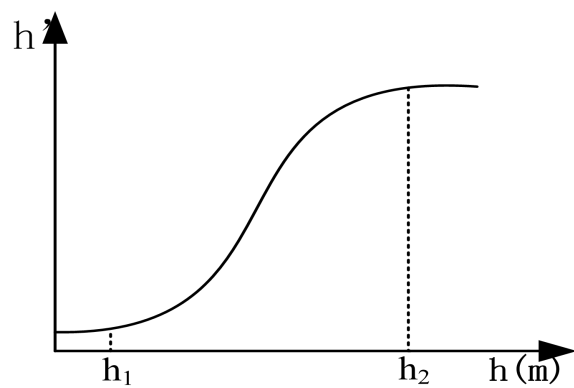

Fig. 3: Function curve of height attribute quantization.

In the operation sections $\mathrm{h} 1$ to $\mathrm{h} 2$, the height of the sector is quantized in accordance with the quantization rule, and the height is quantized to 1,2 . When $h<h 1$, the quantization is $0 ; \mathrm{h}>\mathrm{h} 2$, the quantization of 3 , after the completion of the decision-making table as shown in Table 1.

Table 1: Decision table after quantization of attributes

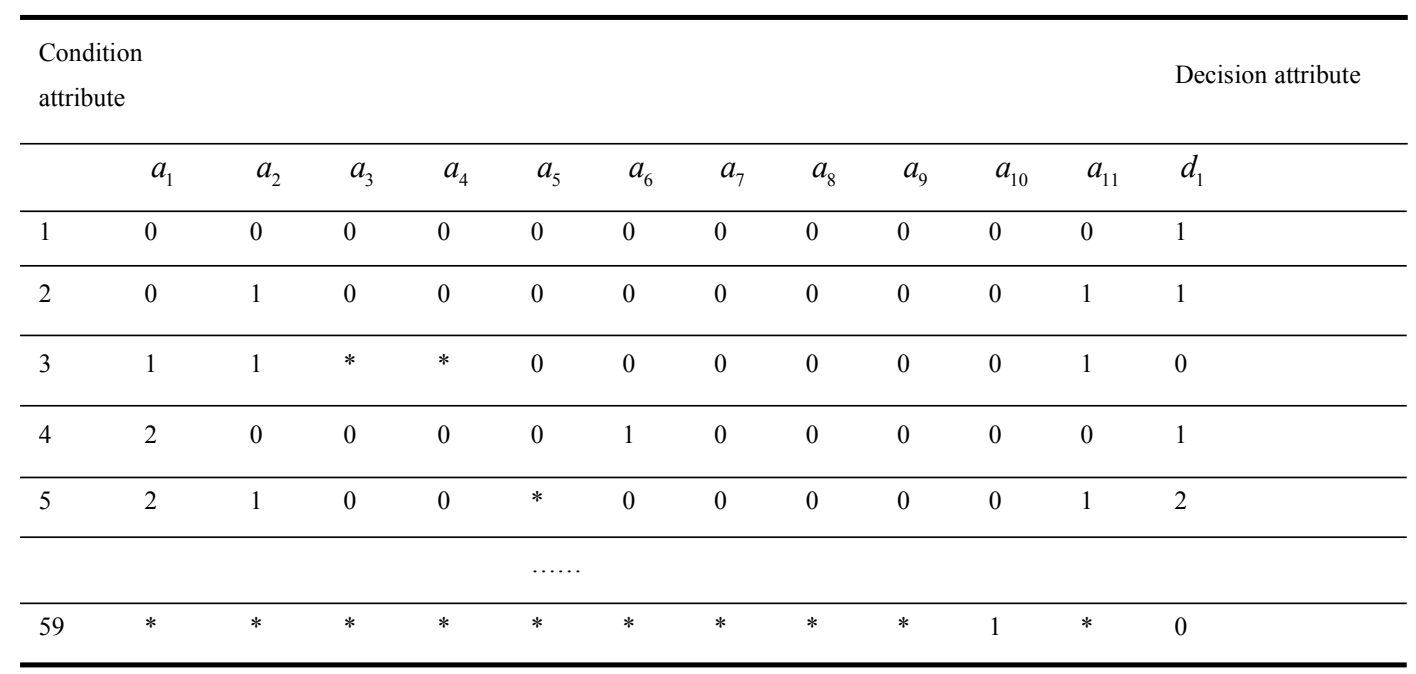

Part of the decision table quantization as shown in Table 1. In table 1 , the condition attributes $\mathrm{C}=\left\{a_{1} a_{2} a_{3} a_{4} a_{5} a_{6} a_{7} a_{8} a_{9} a_{10} a_{11}\right\}$; the decision attributes $\mathrm{D}=\left\{d_{1}\right\}$, among them, $d_{1}$ is the decision state.

\section{Attribute Reduction Based on Attribute Dependence of Rough Set}

In order to verify the comprehensiveness of the condition attribute selection and the rationality of the quantization rule design, the roughness theory is used to reduce the operation attribute, and the job attribute is decomposed by the rough set theory. Redundant attributes, get the simplest decision rules table, so that the design rules can be better verified. According to the definition of attribute set of rough set, in precise mission decision table (table I), condition attribute set consists of $\left\{a_{1} a_{2} a_{3} a_{4} a_{5} a_{6} a_{7} a_{8} a_{9} a_{10} a_{11}\right\}$, decision attribute set by form of $\left\{d_{1}\right\}$. Among it, $\operatorname{POS}_{C}(D)=\operatorname{IND}(C) \cap I N D(D)=U$, this illustrates Table 1 is a fully consistent decision table.

Definition 1 Set $K=(U R), P 、 Q \in R, Q \Rightarrow P$ express knowledge $\mathrm{P}$ depend on $Q$ by the degree of dependence, if and only if

$$
k=\frac{\operatorname{Card}\left(\operatorname{POS}_{p}(Q)\right)}{\operatorname{Card}(U)}
$$


Among it, Card expresses the cardinality of the set, $P O S_{Q}(P)$ expresses $Q$ positive domain of $P$, if $k=1, P$ completely depends on $Q$; if $0<k<1$, P partly depends on $Q$; if $k=1, \mathrm{P}$ is completely independent of $Q$.

The algorithm can be divided into four steps:

Step1: the equivalent of the decision-making class division;

Step2: In turn, the condition attribute set is divided into equivalent classes;

Step3: Solving the intersection, calculation and solution of conditional attribute set equivalence class and decision attribute set equivalence class;

Step4: When a condition attribute set in the 1 , stop the calculation.

According to definition1, $k\left(a_{1} a_{4} a_{5} a_{6} a_{10} a_{11}\right)=1$ expresses that decision attribute set is completely depend on five attributes set $\left\{a_{1} a_{4} a_{5} a_{6} a_{10} a_{11}\right\}$, the result of the reduction of attribute is $\left\{a_{1} a_{4} a_{5} a_{6} a_{10} a_{11}\right\}$.

According to the analysis, it is known that the change of the sensor measurement data and the historical state of the system are much influenced by the state change of the quasi-operational decision system of the $\mathrm{UAV}$, and the change of the decision-making agency has less influence. The decision table after attribute reduction is shown in Table 2.

Table 2: Decision table after attribute reduction

\begin{tabular}{llllllll}
\hline Condition attribute & & & & & \multicolumn{2}{c}{ Decision attribute } \\
\hline & $a_{1}$ & $a_{4}$ & $a_{5}$ & $a_{6}$ & $a_{7}$ & $a_{11}$ & $d_{1}$ \\
\hline 1 & 0 & 0 & 0 & 0 & 0 & 0 & 1 \\
\hline 2 & 0 & 0 & 0 & 0 & 0 & 1 & 1 \\
\hline 3 & 1 & $*$ & 0 & 0 & 0 & 2 & 0 \\
\hline 4 & 1 & 1 & 0 & 1 & 0 & 2 & 1 \\
\hline 5 & 1 & 1 & $*$ & 0 & 0 & 3 & 2 \\
\hline 59 & $*$ & $*$ & $*$ & $*$ & 1 & $*$ & 0 \\
\hline
\end{tabular}

\section{Experiment and Result Analysis}

Based on the background of the International Aircraft Innovation Competition, the decision table generated after reduction is used in the "precision agriculture" system of unmanned aerial vehicle (UAV) and validated on six-rotor aircraft. The aircraft uses the adaptive PID control algorithm and carries the differential GPS and the barometer to carry on the navigation data information fusion, the actual competition scene like Figure 4 shows.

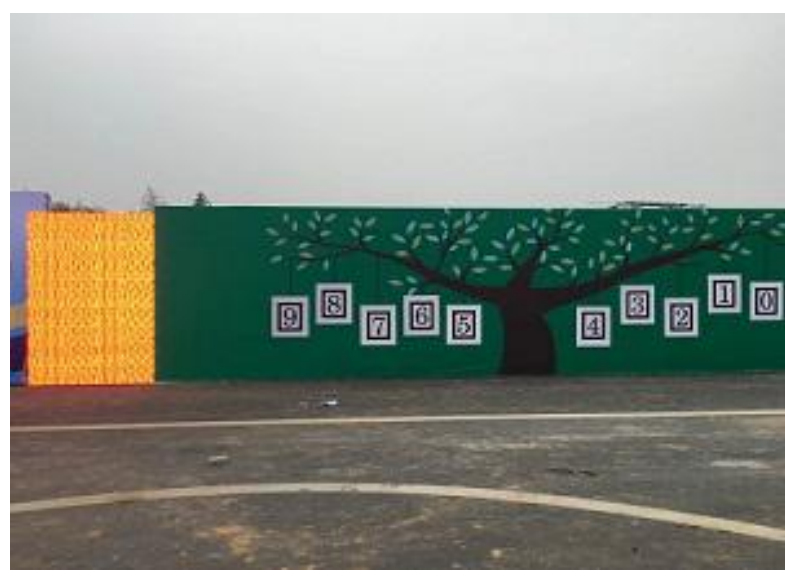

Fig. 4: Arena map of aircraft competition. 
(1) Decision-making experiment after attribute reduction

Using the generated decision rules, the flight experiment of the aircraft is completed. According to the real competition environment, the whole flight trajectory is obtained with six rotors as the carrier platform, as shown in Figure 5.

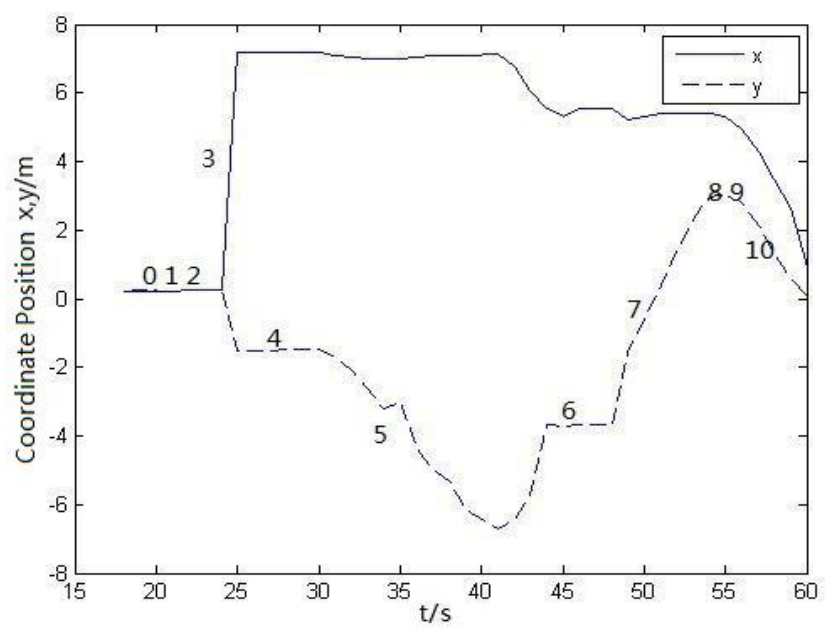

Fig. 5: A complete inkjet flight path map.

Figure 5 shows that, using the decision rules generated in Table 2, the aircraft can achieve complete and accurate changes in the state of each task, from the state 1 - ready to fly to fly to the state - 2 to reach the takeoff height after the fly to the digital area - the state 3 to reach the digital area After the hover recognition number - state 4 , identified after the figure to the drawing area $\mathrm{A}$ - state 5 , the drawing area does not recognize the corresponding figures - state 6, fly to the drawing area B - state 7, identified in the drawing area B digital - State 8, the executing agency inkjet digital - state 9, after the completion of inkjet to fly to the takeoff landing - state 10, the flight process is relatively stable, indicating the choice of attributes and quantitative design is more reasonable to meet task requirements.

(2) Comparison of decision-making after the property increase and decrease

In order to verify the accuracy of the reduction results, some conditional attributes are added or deleted in Table 2, and experiments are carried out on the six rotors to compare the change of decision-making after joining or deleting condition attributes.

(1) Adding the height attribute to the decision table flight path is shown in Figure 6.

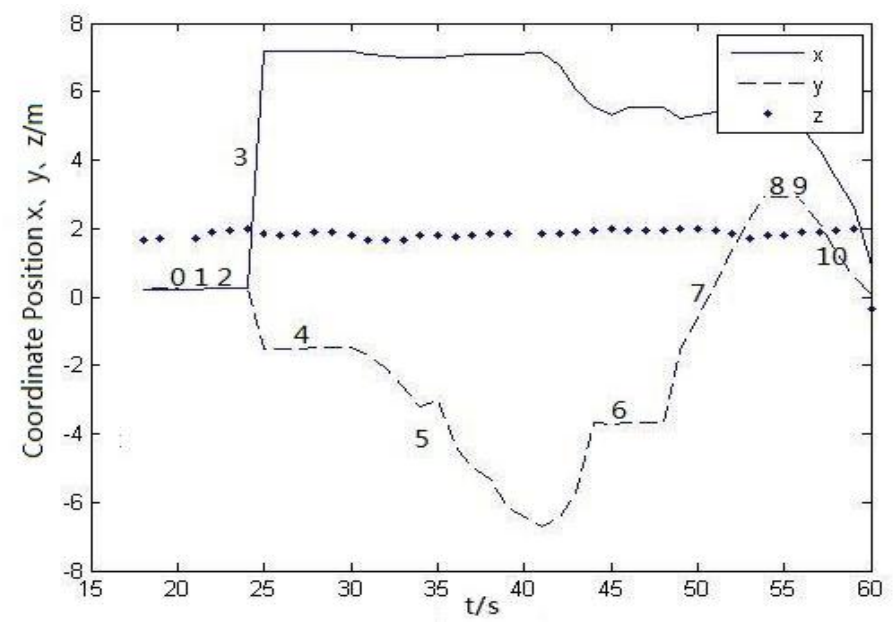

Fig. 6: Flight path map after adding altitude attributes.

As can be seen from Figure 6 and Figure 5, the addition of the altitude attribute has no effect on the switching between states during the flight. Therefore, to remove the altitude attributes has no effect on decision-making classification, belong to unnecessary attributes. 
(2) Removing the digital identification attributes, confidence attributes in decision table, flight path is shown in Figure7.

Digital recognition attribute is used to determine whether the camera to identify the number of information, if the sample space does not contain this property, the UAV in the implementation of the task will lose the "eyes", can not identify and judge the target. The default number has been identified. If no recognition is successful, the UAV will circulate in the drawing area until the flight time ends.

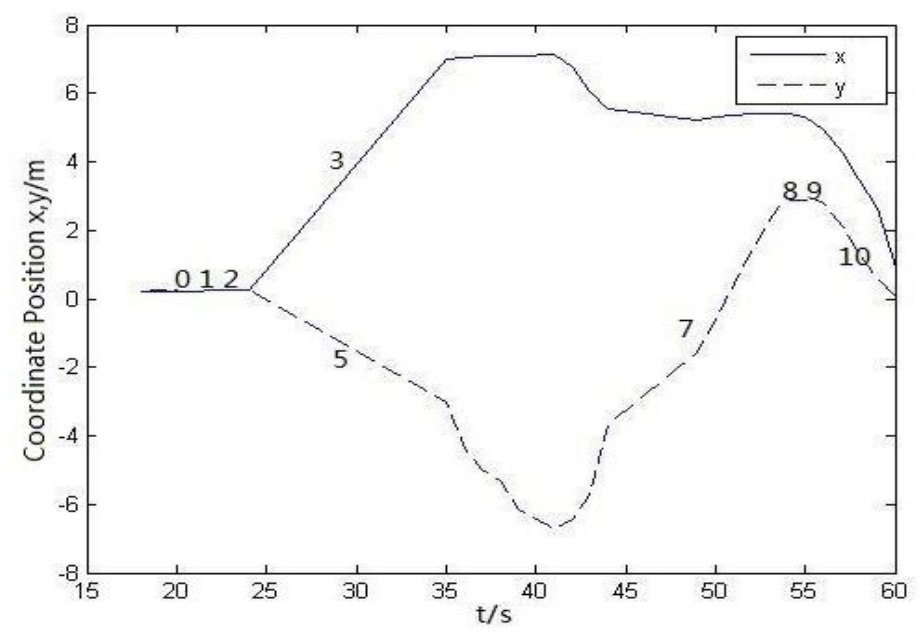

Fig. 7: Flight path map after deleting the numerical identification attributes

It can be seen in Figure7 and Figure 5, after removing the digital identification attributes, the flight process does not appear in the state 4 and state 6 , so the removal of digital identification attributes on the decision classification has a greater impact, it is an important attribute and can not be reduced .

The image confidence attribute is the correct judgment condition of the camera's recognition of the number. If the attribute is ignored, the UAV will not be able to judge whether the recognition result is correct. If the error is identified, the UAV will use the first identified number as the correct number when performing the precise operation. If the accuracy of the image recognition is low, it will have a great impact on the accuracy of the UAV operation.

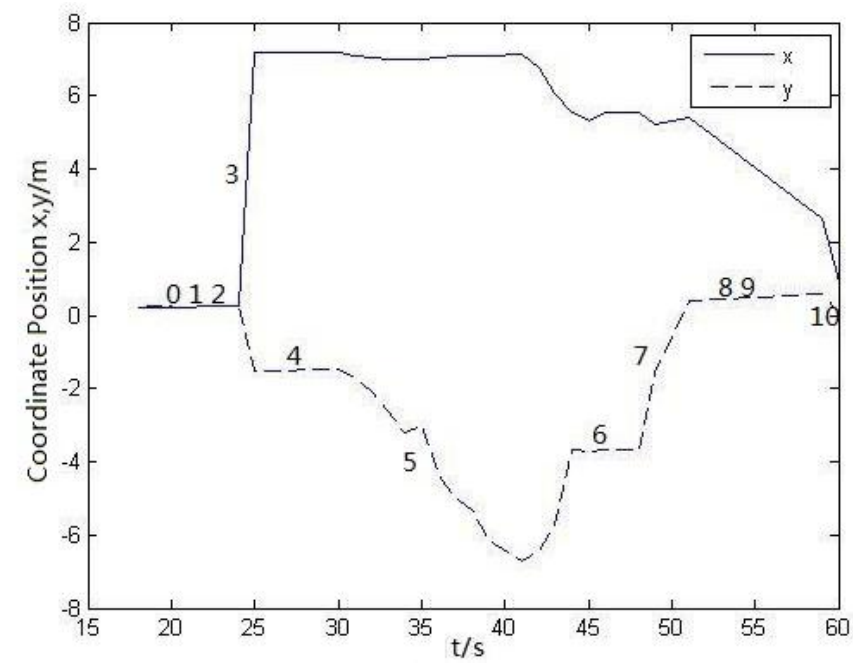

Fig. 8: Flight path after deleting confidence attribute of the image.

It can be seen from Figure 8 and Figure 5, after removing the confidence attribute of the image, state 7 and state 8 do not appear in the course of flight, which have a great influence on decision classification and are important attributes.

(3) The impact of number of operations and the operating time attribute on the decision-making is shown in Figure 9 and Figure 10. 


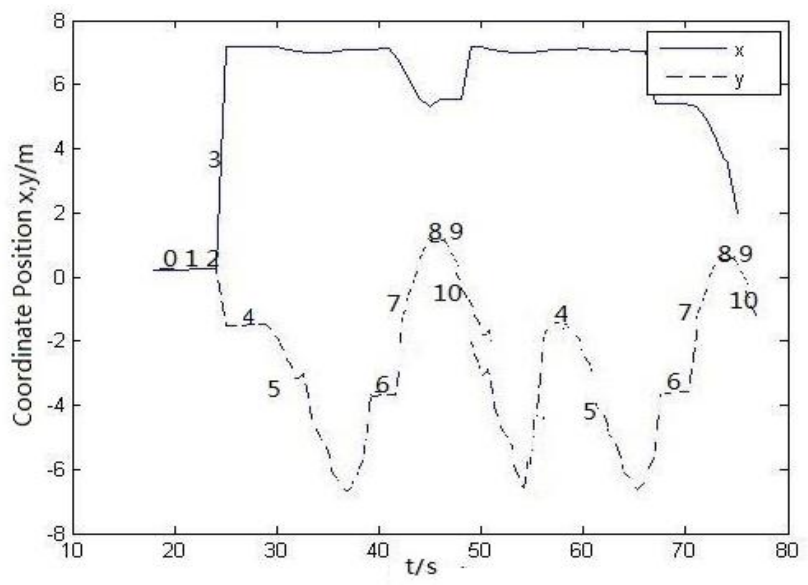

Fig. 9: Flight trajectory for 2 operations.

It can be seen from Figure 9 and Figure 5, when the second task is executed, the aircraft will return to state 4, which is consistent with the change of the state of the execution of a task and has no great influence on the decision. In the state 8 , it returns to the normal position, indicating that the flight process has strong robustness.

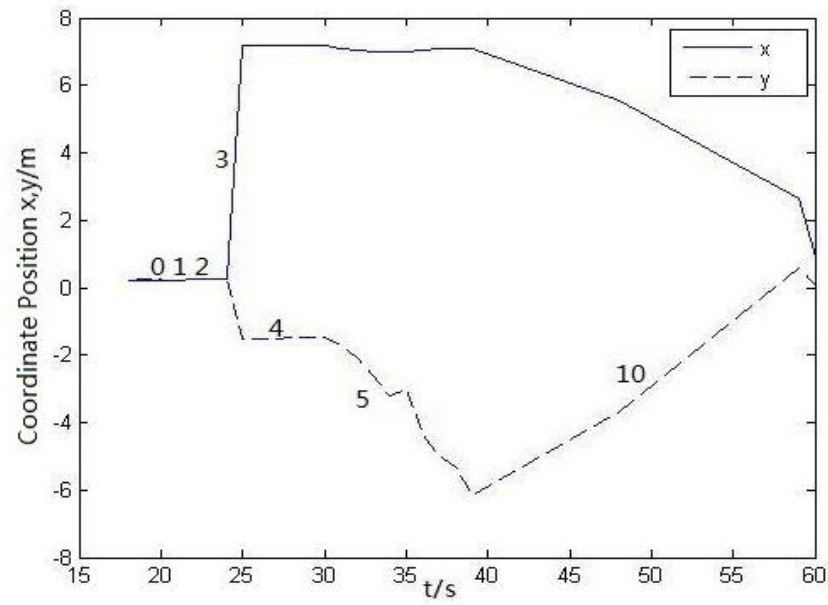

Fig. 10: Flight trajectory for the arrival time.

It can be seen from Figure10, when the aircraft arrives at the time of flight, the aircraft will return to the origin state. It has a great influence on the classification of decision making and belongs to important attributes and can not be removed.

(4) The impact of historical state attributes on decision making is shown in Figure 11.

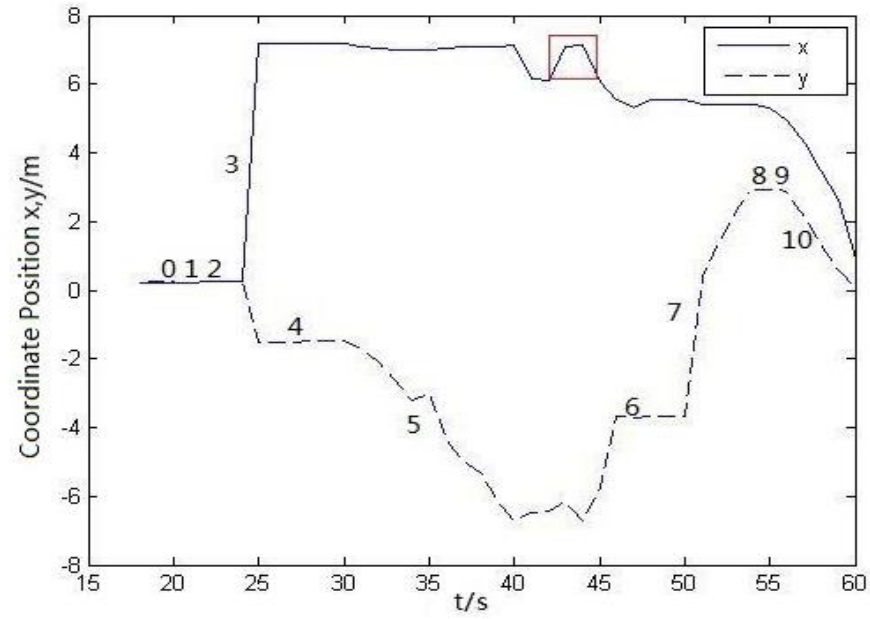

Fig. 11: Flight trajectory in abnormal state 
In Figure 11, when the aircraft is disturbed or deviated from the normal state during flight, the aircraft can return to normal flight due to the limitation of the historical state, so the addition of the historical state attribute is very necessary.

According to the analysis of experimental flight trajectories, we can see that the decision table after attribute reduction can achieve precise operation. Therefore, when the condition attribute is selected, the data of the sensor and the historical state data, which influence the decision state more greatly, the influence of change of the actuator is relatively small.

\section{Conclusion}

The method of decision rule generation for UAVs during precise operation is studied. The design rules for the attributes of the operation space are put forward emphatically; Based on the distribution density of the operating condition attribute range, a fine quantization method based on arctangent characteristics is proposed; And the attribute reduction algorithm based on dependency is adopted to remove the redundant attribute and unrelated attribute, generating the decision rule. The experimental results show that the proposed design rules and quantification method can achieve the accurate completion of the task and ensure the stability of the task. It provides a new method for UAV decision rules generation.

\section{References}

[1] CHEN, H L. Research on Machine Learning Methods for Intelligent Decision-Making [D].Jilin university,2012(5).

[2] SUN, H R.A Method of Machine Learning and The Design of Inference Engine based on Decision Tree [D]. Changchun Industrial University,2014(4).

[3] DENG,B B. Information-gain based quantization algorithm and its application in decision tree study[D].Guangdong Industrial University,2016(5).

[4] LI,H,YAN D Q,HAN L.A New Algorithm for Quantization of Continuous Attributes Based on Rough Set Theory[J].Computer Applications and Research,2010(1),27(1):77-78.

[5] ZHANG L. Research on autonomous decision-making technology of UCAV[J].Aeronautical Science \& Technology,2014,25(05):49-53.

[6] REN,J,GAO,Y. Stochastic multi-criterion decision-making method based on interval operation[J]. Systems Engineering and Electronics,2010,32(2):308-312.

[7] HUANG,Z H,HU B Q. Research Progress of Fuzzy Rough Set Theory[J].Fuzzy Systems and Mathematics,2005,19(4):125-134.

[8] Qinglin, Guo, Kehe, Wu, Wei, Li. Fault forecast and diagnosis of steam turbine based on fuzzy rough set theory[C]. 2nd International Conference on Innovative Computing, Information and Control, ICICIC 2007,2008[7].

[9] Chen, Fu Hsiang, Chi, Der-Jang, Kuo, Chun-Yi. Using rough set theory and decision trees to diagnose enterprise distress - Consideration of corporate governance variables[C]. 10th International Conference on Intelligent Computing, ICIC 2014,2014(8).

[10]Liu, Xing wen, Wang, Dian hong. An improved algorithm for oblique decision tree classification based on rough set theory[J]. Journal of Computational Information Systems,2011(11).

[11]ZHANG,Q H,HU,R D,YAO,L Y,XIE W Y. Risk DTRS attribute reduction based on attribute importance[J].Control and decision-making,2016(7),31(7):1199-1205. 